\title{
Comparison of efficacy of fluticasone propionate versus clarithromycin for postoperative treatment of different phenotypic chronic rhinosinusitis: a randomized controlled trial* $^{*}$
}

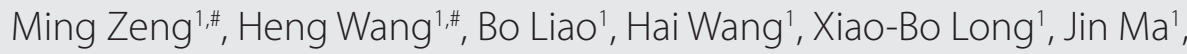 \\ Jin-Xin Liu', Ping-Ping Cao', Qin Ning², Zheng Liu' \\ ' Department of Otolaryngology-Head and Neck Surgery, Tongji Hospital, Tongji Medical College, Huazhong University of \\ Science and Technology, Wuhan, China \\ ${ }^{2}$ Department of Infectious Disease, Institute of Infectious Disease, Tongji Hospital, Tongji Medical College, Huazhong \\ University of Science and Technology, Wuhan, China
}

Rhinology 57: 2, $101-109,2019$

https://doi.org/10.4193/Rhin17.226

*Received for publication:

October 17, 2017

Accepted: July 18, 2018

Background: Chronic rhinosinusitis (CRS) can be divided to CRS without nasal polyps (CRSsNP) and eosinophilic and non-eosinophilic CRS with nasal polyps (CRSwNP). There is little evidence on the efficacy of glucocorticoids and macrolides in different phenotypic patients. The aim of this study was to compare the benefit of glucocorticoids and macrolides following endoscopic sinus surgery (ESS) in different phenotypic CRS.

Methods: This study was a prospective single-blind comparative effectiveness trial. A total of 187 Chinese patients with CRS were stratified to CRSsNP and eosinophilic and non-eosinophilic CRSwNP group and then randomized to receive fluticasone propionate nasal spray at $200 \mu \mathrm{g}$ or clarithromycin tablet at $250 \mathrm{mg}$ once daily for 3 months after ESS. Oral prednisone was given as a rescue therapy after the stop of study medication. Patients were assessed before ESS and 1, 3, 6 and 12 months after dosing. Symptom severity was scored by patients using visual analog scale method and endoscopic findings were scored by the senior physician blinded to treatment according to European Position Paper on Rhinosinusitis and Nasal polyps 2012.

Results: The total and individual symptom scores, and total and individual endoscopic domain scores were reduced significantly after ESS in both medication groups, whereas no significant difference was observed for two medications at most follow-up visits in each subtype of CRS. No difference in the frequency of subjects with rescue therapy or refractory CRS was found between two medication groups either.

Conclusions: We could not show significant difference of effect between fluticasone propionate and clarithromycin in the postoperative treatment for CRSsNP and eosinophilic and non-eosinophilic CRSwNP patients.

Key words: chronic rhinosinusitis, clarithromycin, fluticasone propionate, nasal polyps, postoperative treatment

\section{Introduction}

Chronic rhinosinusitis (CRS) is a multifactorial inflammatory disorder that causes various symptoms including nasal obstruction, rhinorrhea, olfactory dysfunction, facial pain, and headache (1,

${ }^{2}$. Endoscopic sinus surgery (ESS) is usually recommended for patients who do not response well to conservative treatments (3). Although surgery is effective to alleviate symptoms and improve the quality of life of CRS patients, a followed long-term postoperative medical treatment is indispensable ${ }^{(2,3)}$.

Current European and American guidelines recommend glucocorticoids and antibiotics as the primary pharmacotherapeutic approaches for CRS before and after ESS ${ }^{(2,3)}$. However, there are a number of CRS patients not responding well to glucocorticoid treatment and conflicting results exist regarding the efficacy of long-term, low-dose macrolide treatment ${ }^{(4-6)}$. One potential reason is that CRS is a heterogeneous group of disorders unified 
by similar clinical presentations and its phenotypes and/or endotypes may be important factors determining the efficacy of treatments ${ }^{(7-9)}$.

CRS can be broadly defined as either with nasal polyps (CRSwNP) or without nasal polyps (CRSsNP). In Caucasians, CRSsNP presents a type 1 response, whereas CRSwNP is dominated by eosinophilic inflammation with a skewed type 2 response ${ }^{(10)}$. However, CRS is more heterogeneous than this clinical classification. In East Asians, a considerable number of CRSwNP patients don't demonstrate eosinophilic inflammation (11-13), and non-eosinophilic CRSwNP is characterized by a type 1/type 17 cytokine milieu and a more neutrophilic inflammation ${ }^{(10-15)}$. Our previous self-controlled study with a small sample size reported that although oral prednisone was able to suppress the eosinophilic inflammation, it was less efficient to inhibit the type 17 responses and neutrophilic inflammation in Chinese CRSwNP patients without surgery ${ }^{(16)}$. Wallwork et al. found that longterm low-dose macrolide treatment was only efficient for CRSsNP patients without elevated serum IgE levels ${ }^{(4)}$. We demonstrated that long-term low dose macrolide treatment was more effective for CRSsNP patients with higher neutrophilic inflammation ${ }^{(17)}$. We therefore hypothesized that glucocorticoids may be more effective on eosinophilic CRS, whereas macrolides are better to be tailored for non-eosinophilic CRS. However, there are few studies comparing the efficacy of glucocorticoids and macrolides in the treatment of different phenotypic CRS. In this study, we conducted a prospective two-arm, randomized, and controlled trial to compare the benefit of fluticasone propionate nasal spray and clarithromycin tablet following ESS in different phenotypic CRS defined by the presence or absence of nasal polyps and eosinophilic inflammation, i.e., CRSsNP, eosinophilic CRSwNP, and non-eosinophilic CRSwNP.

\section{Materials and methods}

\section{Patients}

Patients meeting the CRS diagnostic criteria outlined in the European Position Paper on Rhinosinusitis and Nasal polyps (EP3OS) 2012 definition were enrolled ${ }^{(3,18)}$. All the patients had ongoing symptoms after initial attempts on medical treatments, including nasal or oral steroids and nasal saline irrigation ${ }^{(3,19)}$. The atopic status was evaluated using the skin prick test with a standard panel of common inhalant allergens common in our region ${ }^{(20)}$. The diagnosis of allergic rhinitis was based on the concordance between a typical history and the skin prick test results ${ }^{(20)}$. The diagnosis of asthma was made according to Global Initiative for Asthma guideline ${ }^{(21,22)}$. The detailed inclusion and exclusion criteria are shown in Table S1 in the Online Supplement. This study was approved by the Ethics Committee of Tongji Hospital and conducted with written informed consents from all participants.

\section{Study design}

This study was designed as a monocenter, prospective two-arm, randomized, single-blind, and comparative effectiveness trial (ClinicalTrials.gov Identifier: NCT02182492). ESS was performed by the senior surgeon (Z.L.) in accordance with the extent of the disease as described by Stammberger and Kennedy ${ }^{(23,24)}$. The surgery extent was determined based on the extent of sinusitis as revealed by sinus computed tomographic (CT) images and intraoperative judgement of the surgeon ${ }^{(25)}$. CT scans were graded in line with Lund-Mackay CT scoring system and total bilateral CT scores were analyzed ${ }^{(3,26)}$. Polyp tissues from CRSwNP patients were obtained during surgery and processed for histology study. CRSwNP was further classified as eosinophilic when percent tissue eosinophils exceeded $10 \%$ of total infiltrating cells as defined by our previous report ${ }^{(11)}$. This cut-off was calculated as twice of the standard deviation of the mean of eosinophil percentage in controls ${ }^{(11)}$. Randomization was stratified according to CRS phenotypes. One week after surgery, CRSsNP, eosinophilic CRSwNP, and non-eosinophilic CRSwNP patients were randomly assigned to fluticasone propionate or clarithromycin group using a random number table separately. In fluticasone propionate group, patients were given fluticasone propionate nasal spray (Glaxo Wellcome, S.A., Burgos, Spain), $200 \mu \mathrm{g}$ once daily for 3 months. In clarithromycin group, patients received $250 \mathrm{mg}$ tablet of clarithromycin (Abbot China, Shanghai, China) once daily for 3 months. Patients were evaluated before ESS and 1, 3, 6 and 12 months after dosing with the study medication. The pre-operative evaluation was set as "baseline". Compliance was checked by weighting study drug bottles in fluticasone propionate group and counting the number of pills left in clarithromycin group ${ }^{(27,28)}$. Meanwhile, all the subjects were prescribed manual nasal irrigation device together with $0.9 \%$ sodium chloride solutions ( $250 \mathrm{~mL}$ in a transparent bottle). Patients performed nasal saline irrigation ( $250 \mathrm{~mL}$ twice a day) for 1 year. Each irrigation device was replaced with a new one every 1-2 months. The compliance of nasal saline irrigation was checked by examining and/or weighting drug bottles in both groups. During the study period, additional visits to the physician were allowed. Short-course (1-2 weeks) of intranasal steroid treatment after stop of study medication was permitted, but only under the situation of exacerbation of mild symptoms that usually followed common cold ${ }^{(29)}$. If polyps and/or the moderate to severe clinical symptoms of CRS were relapsed and/or not controlled after the stop of study medication, rescue therapy with oral prednisone tablets (30 mg daily on days $1-5,20 \mathrm{mg}$ daily on days $6-10,10 \mathrm{mg}$ daily on days $11-15$, and $5 \mathrm{mg}$ daily on days $16-20$ ) was prescribed ${ }^{(30)}$. For intranasal steroid and rescue oral steroid treatment after stop of study medication, the clinical symptom severity was determined by visual analog scale (VAS) scores of overall burden of symptoms: mild = VAS 0-3; moderate $=$ VAS $>3-7$; severe $=$ VAS $>7-10$, in accordance with the recom- 


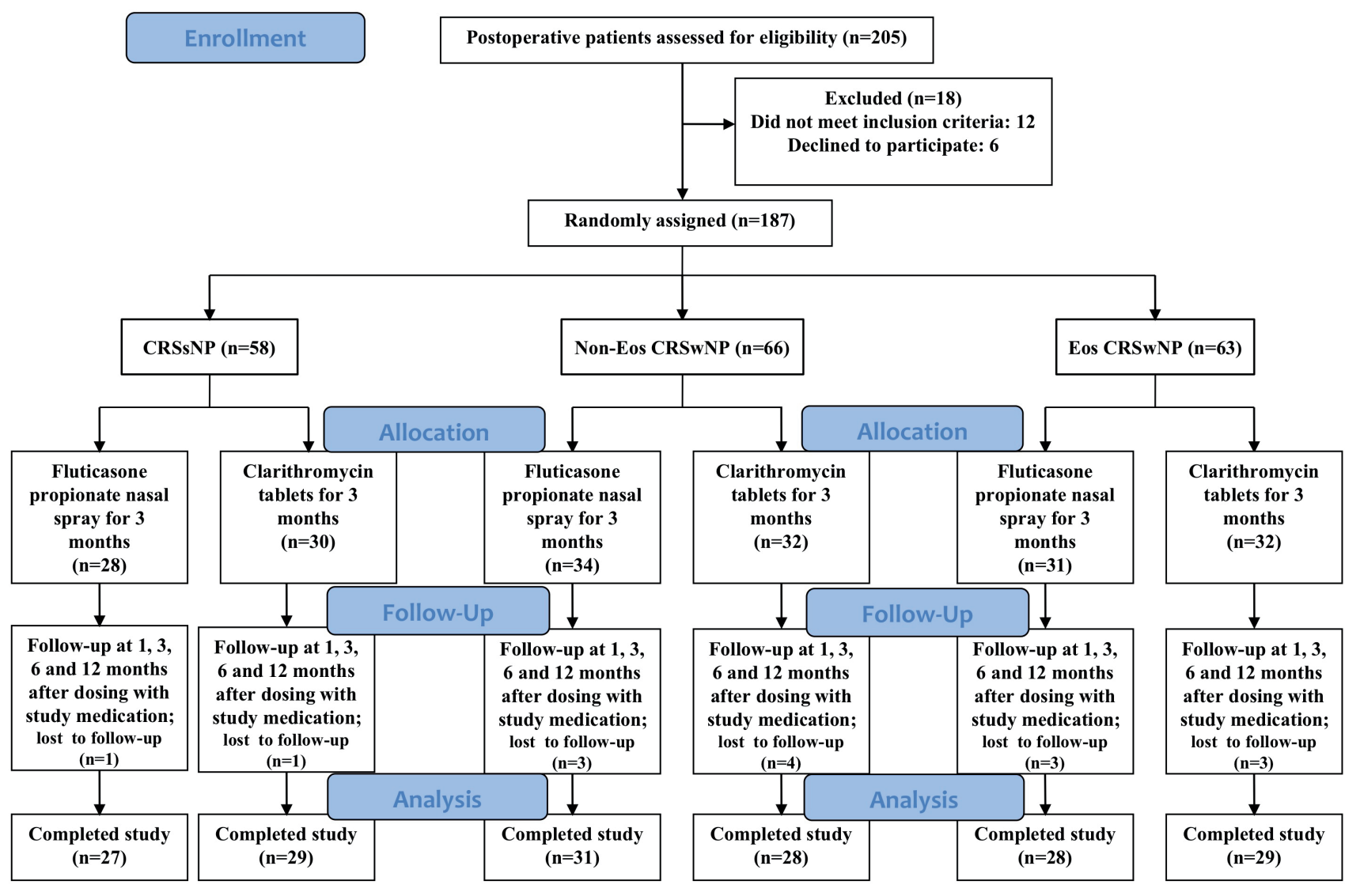

Figure 1. Study flow chart. CRSsNP, chronic rhinosinusitis without nasal polyps; Non-Eos CRSwNP, non-eosinophilic chronic rhinosinusitis with nasal polyps; Eos CRSwNP, eosinophilic chronic rhinosinusitis with nasal polyps.

mendation of EP3OS $2012^{(3,31)}$. VAS scoring was performed as described below.

\section{Outcome measures}

All of the patients were assessed by using symptom questionnaire and nasal endoscopy just before ESS (baseline) and 1, 3, 6 and 12 months after dosing with the study medication. Subjective symptoms were scored by patients on a VAS of $0-10$, with 0 being "no complaint whatsoever" and 10 being "the worst imaginable complaint." Five major symptoms were focused on: nasal obstruction, rhinorrhea, loss of sense of smell, facial pain or pressure, and headache ${ }^{(3,31)}$. A total VAS symptom score was calculated based on the sum of VAS scores of these five symptom domains ${ }^{(3,31)}$. In addition, every patient was asked to rate his/her overall burden of CRS symptoms. Symptoms were scored by patients and recorded by investigators (J.M. and J.X.L.) who were blinded to the treatment in the record forms.

Nasal endoscopic evaluation was performed by the senior investigator (Z.L.) who remained blinded to the treatment. Endoscopic scoring was performed according to EP3OS 2012 (poly size: 0 , absence of polyps; 1 , polyps in middle meatus only; 2 , polyps beyond middle meatus but not blocking the nose completely; and 3, polyps completely obstructing the nose; discharge: 0 , no discharge; 1 , clear thin discharge; 2 , thick purulent discharge; edema: 0 , no edema; 1 , mild edema; 2 , severe edema; crusting: 0 , no crusting; 1 , mild crusting; 2 , severe crusting; scarring: 0 , no scarring; 1 , mild scarring; 2 , severe scarring) ${ }^{(3,32)}$. Each side was graded separately, and the scores from both sides were added to determine the overall scores for a particular domain. A total endoscopy score was calculated based on the sum of scores of these endoscopic domains.

The primary outcome measure was total VAS symptom scores. The secondary outcome measures included individual symptom VAS scores, overall symptom burden scores, total endoscopic scores, and individual endoscopic domain scores. Subjects were considered as refractory cases if they still had bothersome symptoms even with rescue medication ${ }^{(3,33)}$. The numbers of oral steroid courses, the time from the 3-month follow-up visit to the first course of rescue oral steroid therapy in patients who received rescue therapy, the proportions of patients receiving nasal glucocorticoids and rescue oral prednisone treatment, and the proportions of refractory cases were also evaluated at 12-month post-dosing follow-up visit. 
Table 1. Patients' demographic and clinical characteristics.

\begin{tabular}{|c|c|c|c|c|c|c|c|c|c|}
\hline & \multicolumn{3}{|c|}{ Fluticasone propionate group } & \multicolumn{3}{|c|}{ Clarithromycin group } & \multirow[t]{2}{*}{ P* } & \multirow[t]{2}{*}{$\mathbf{P}^{+}$} & \multirow[t]{2}{*}{$\mathbf{p} \neq$} \\
\hline & CRSsNP & $\begin{array}{l}\text { Non-Eos } \\
\text { CRSwNP }\end{array}$ & Eos CRSwNP & CRSsNP & $\begin{array}{l}\text { Non-Eos } \\
\text { CRSwNP }\end{array}$ & Eos CRSwNP & & & \\
\hline Subject, $\mathrm{n}$ & 28 & 34 & 31 & 30 & 32 & 32 & - & - & - \\
\hline Sex, male, n (\%) & $16(57.1)$ & 19 (55.9) & $20(64.5)$ & $16(53.3)$ & 15 (46.9) & $21(65.6)$ & 0.98 & 0.63 & 1.00 \\
\hline Age (years), median (IQR) & $\begin{array}{c}37 \\
(31.5-44.5)\end{array}$ & $\begin{array}{c}43 \\
(29.8-49)\end{array}$ & $\begin{array}{c}45 \\
(36.5-51)\end{array}$ & $\begin{array}{c}35.5 \\
(25-42.5)\end{array}$ & $\begin{array}{c}41 \\
(32-54.3)\end{array}$ & $\begin{array}{c}43.5 \\
(32.5-53)\end{array}$ & 0.55 & 0.79 & 0.86 \\
\hline Smoking", n (\%) & $11(39.3)$ & $7(20.6)$ & $10(32.3)$ & $9(30)$ & $5(15.6)$ & $10(31.3)$ & 0.64 & 0.84 & 1.00 \\
\hline Patients with atopy, n (\%) & $5(17.9)$ & $8(23.5)$ & $9(29)$ & $6(20)$ & $6(18.8)$ & $12(37.5)$ & 1.00 & 0.86 & 0.60 \\
\hline Patients with AR, n (\%) & $2(7.1)$ & $4(11.8)$ & $8(25.8)$ & $4(13.3)$ & 5 (15.6) & $11(34.4)$ & 0.73 & 0.92 & 0.59 \\
\hline Patients with asthma, n (\%) & $0(0)$ & $0(0)$ & $2(6.5)$ & $0(0)$ & $1(3.1)$ & $4(12.5)$ & 1.00 & 1.00 & 0.70 \\
\hline $\begin{array}{l}\text { Patients with prior sinus } \\
\text { surgery, } \mathrm{n}(\%)\end{array}$ & $5(17.9)$ & $8(23.5)$ & $10(32.3)$ & $7(23.3)$ & $6(18.8)$ & $10(31.3)$ & 0.85 & 0.86 & 1.00 \\
\hline $\begin{array}{l}\text { Bilateral CT score, median } \\
\text { (IQR) }\end{array}$ & $9(6-11)$ & $16(12.8-19)$ & $15(12-18)$ & $8(6.8-10)$ & $16.5(14-19)$ & $16(14.3-19)$ & 0.85 & 0.70 & 0.50 \\
\hline
\end{tabular}

CRSsNP, chronic rhinosinusitis without nasal polyps; Non-Eos CRSwNP, non-eosinophilic chronic rhinosinusitis with nasal polyps; Eos CRSwNP, eosinophilic chronic rhinosinusitis with nasal polyps; AR, allergic rhinitis; IQR, interquartile range. P values, * ${ }^{*}$ comparison of two different medication groups in CRSsNP patients; ${ }^{\dagger}$ comparison of two different medication groups in Non-Eos CRSwNP patients; ${ }^{\ddagger}$ comparison of two different medication groups in Eos CRSwNP patients. "Active smoking history $>1$ year.

\section{Adverse events}

Adverse events were recorded throughout the study including asthma exacerbation, fever, headache, nausea, diarrhea, nasal bleeding, and nasal irritation, etc.

\section{Statistical analysis}

A preplanned pilot study with 36 CRS patients was undertaken to estimate the sample size by using total VAS symptom scores as the primary parameter. The pilot study discovered a 3-unit of mean difference in total VAS symptom scores at 3-month after dosing between two medications. We therefore estimated that 25 subjects per group would be required to detect a 3-unit of mean difference (combined SD, 3.2) with a power equal to $90 \%$ and a 2 -tailed a value of $5 \%{ }^{(34)}$. Considering a possible loss of $20 \%$ of patients at follow-up, we planned to recruit 30 participants in each study group.

The statistical analysis of efficacy was based on subjects who completed the study. Since variables were not normally distributed as analyzed by Kolmogorov-Smirnov test or ShapiroWilk test, Mann-Whitney U 2-tailed test was used to analyzed baseline values, the numbers of oral steroid courses, and the time from the 3-month follow-up visit to the first course of rescue therapy in patients who received rescue therapy between groups. Chi-square test was applied to compare the differences in proportions between groups. Changes in clinical parameters after medical treatments were evaluated by repeated-measures ANOVA analyses after log-transformation of data. Differences were considered to be statistically significant if $P$ values were less than 0.05. Bonferroni correction was applied to the $P$ values for multiple comparisons. Statistical analyses were performed with SPSS for Windows, Version 19.0 (SPSS, Inc., Chicago, IL, USA).

\section{Results}

Patient's clinical characteristics

Overall, 205 postoperative patients were assessed for eligibility. Twelve patients did not meet inclusion criteria and 6 patients declined to participate. A total of 187 patients met the study eligibility criteria. Those patients were stratified into CRSsNP and non-eosinophilic and eosinophilic CRSwNP group, and were then randomly allocated to fluticasone propionate or clarithromycin group (Figure 1). Seven patients in fluticasone propionate group and 8 patients in clarithromycin group dropped out because of nonadherence (Figure 1). There was no significant difference of total CT scores between two medication groups in any of the phenotypic CRS groups (Table 1), and therefore the surgery extent was comparable between two medication groups. No significant difference of any other clinical characteristic was identified between two medication groups either (Table 1). Patient compliance with fluticasone propionate, clarithromycin, and nasal saline irrigation was over $95 \%$, and no significant difference was found between two medication groups in any of the phenotypic CRS groups (data not shown). 

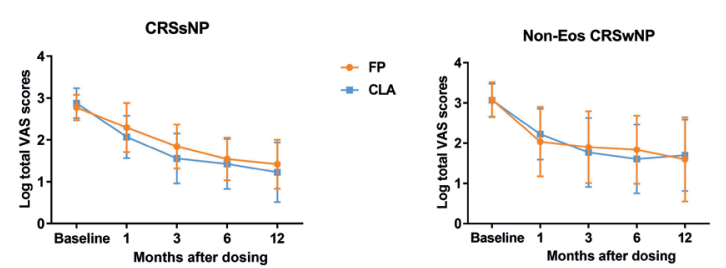

$\rightarrow$ FP
- CLA
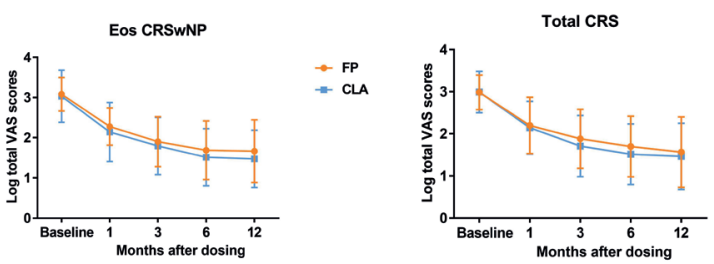

$\rightarrow$ FP
- CLA

Figure 2. Comparison of the effect of post-operative fluticasone propionate (FP) and clarithromycin (CLA) treatment on total visual analog scale (VAS) symptom scores in different phenotypic chronic rhinosinusitis (CRS) as well as whole CRS group. Log-transformed data are expressed as means \pm SD. The pre-operative evaluation was set as "baseline". CRSsNP, CRS without nasal polyps; Non-Eos CRSwNP, non-eosinophilic CRS with nasal polyps; Eos CRSwNP, eosinophilic CRS with nasal polyps.
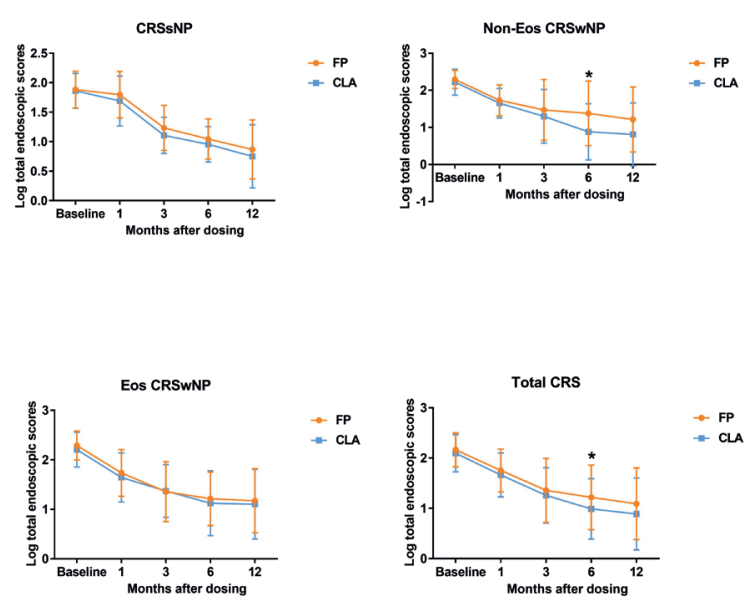

Figure 3. Comparison of the effect of post-operative fluticasone propionate (FP) and clarithromycin (CLA) treatment on total endoscopic scores in different phenotypic chronic rhinosinusitis (CRS) as well as whole CRS group. Log-transformed data are expressed as means $\pm \mathrm{SD}$. The preoperative evaluation was set as "baseline". CRSsNP, CRS without nasal polyps; Non-Eos CRSwNP, non-eosinophilic CRS with nasal polyps; Eos CRSwNP, eosinophilic CRS with nasal polyps. ${ }^{*} \mathrm{P}<0.05$.

\section{VAS symptom scores}

We compared the effect of fluticasone propionate and clarithromycin on postoperative symptom and endoscopic appearance in each particular phenotypic CRS group as well as total CRS patients. No significant difference in baseline total VAS symptom scores between fluticasone propionate and clarithromycin group was found in each subtype of CRS and total CRS irrespective of phenotypes (Figure 2). The total VAS symptom scores were reduced significantly after ESS in both medication groups in all the phenotypic CRS. Surprisingly, no significant difference in total VAS symptom scores was observed between fluticasone propionate and clarithromycin group in all phenotypic CRS as well as whole CRS group at 1, 3, 6, and 12-month post-dosing follow-up visits (Figure 2). As to each individual symptom domain and overall burden of symptoms, no significant difference in baseline VAS scores was found between subjects with fluticasone propionate and clarithromycin treatment in each subtype of CRS and whole CRS group either (Figures S1-S4 in the Online Supplement). Moreover, VAS scores of those individual symptoms and overall burden of symptoms were reduced significantly after ESS in both medication groups in all phenotypic CRS and whole CRS group (Figures S1-S4 in the Online Supplement). Again, we failed to find any significant difference in VAS scores of each symptom domain or overall burden of symptoms between fluticasone propionate and clarithromycin treatment group in CRSsNP, eosinophilic CRSwNP or non-eosinophilic CRSwNP subjects, or total CRS group irrespective of phenotypes at any of the post-dosing follow-up visits (Figures S1-S4 in the
Online Supplement).

\section{Endoscopic scores}

Similar to VAS symptom scores, no significant difference in baseline total endoscopic scores or scores of each endoscopic domain between fluticasone propionate and clarithromycin treatment group could be found in each subtype of CRS as well as whole CRS group (Figure 3 and Figures S5-S8 in the Online Supplement). The total endoscopic scores were reduced significantly right after ESS in both medication groups (Figure 3). No significant difference in total endoscopic scores was found between fluticasone propionate and clarithromycin treatment group in subjects with CRSsNP and eosinophilic CRSwNP at any of the follow-up visits (Figure 3). In non-eosinophilic CRSwNP group and whole CRS group, patients treated with fluticasone propionate demonstrated higher total endoscopic scores at 6-month, but not 12-month, post-dosing follow-up visit compared with those treated with clarithromycin (Figure 3). As to each particular endoscopic domain, we found that the scores of discharge and polyps were significantly decreased as early as 1 month after dosing of the study medication, whereas the scores of edema were decreased 3 months after dosing (Figures S5-S8 in the Online Supplement). In contrast, no significant change of scarring and crusting scores was found at 12-month followup visit after dosing compared with baseline scores (Figures S5-S8 in the Online Supplement). No significant difference in the scores in any of the endoscopic domains was discovered between fluticasone propionate and clarithromycin treatment 
Table 2. Percentages of refractory cases and patients receiving nasal glucocorticoids and rescue oral prednisone therapy after stop of study medications.

\begin{tabular}{|c|c|c|c|c|c|c|c|c|c|c|c|}
\hline & \multicolumn{4}{|c|}{ Fluticasone propionate group } & \multicolumn{4}{|c|}{ Clarithromycin group } & \multirow[t]{2}{*}{ P* } & \multirow[t]{2}{*}{$\mathbf{P}^{+}$} & \multirow[t]{2}{*}{$\mathbf{P} \neq$} \\
\hline & $\begin{array}{c}\text { Total, } \\
\mathbf{n}\end{array}$ & $\begin{array}{c}\text { Refractory } \\
\text { case, } \\
\text { n (\%) }\end{array}$ & $\begin{array}{c}\text { Rescue } \\
\text { therapy, } \\
\text { n (\%) }\end{array}$ & $\begin{array}{l}\text { Nasal } \\
\text { glucocorti- } \\
\text { coids, } \\
\text { n (\%) }\end{array}$ & $\begin{array}{c}\text { Total, } \\
\mathbf{n}\end{array}$ & $\begin{array}{c}\text { Refractory } \\
\text { case, } \\
\text { n (\%) }\end{array}$ & $\begin{array}{c}\text { Rescue } \\
\text { therapy, } \\
\text { n (\%) }\end{array}$ & $\begin{array}{c}\text { Nasal } \\
\text { glucocorti- } \\
\text { coids, } \\
\text { n (\%) }\end{array}$ & & & \\
\hline CRSsNP & 27 & $5(18.5)$ & $10(37)$ & $8(29.6)$ & 29 & $5(17.2)$ & $11(37.9)$ & $7(24.1)$ & 0.92 & 0.96 & 0.87 \\
\hline Non-Eos CRSwNP & 31 & 7 (22.6) & $19(61.3)$ & $15(48.4)$ & 28 & $5(17.9)$ & $15(53.6)$ & $11(39.3)$ & 0.71 & 0.76 & 0.66 \\
\hline Eos CRSwNP & 28 & $7(25)$ & $14(50)$ & $10(35.7)$ & 29 & $8(27.6)$ & $15(51.7)$ & $13(44.8)$ & 0.87 & 0.94 & 0.67 \\
\hline Total CRS & 86 & $19(22.1)$ & $43(50)$ & $33(38.4)$ & 86 & $18(20.9)$ & $41(47.7)$ & $31(36)$ & 0.88 & 0.86 & 0.75 \\
\hline
\end{tabular}

CRSsNP, chronic rhinosinusitis without nasal polyps; Non-Eos CRSwNP, non-eosinophilic chronic rhinosinusitis with nasal polyps; Eos CRSwNP, eosinophilic chronic rhinosinusitis with nasal polyps; CRS, chronic rhinosinusitis. P values, *comparison of percentage of refractory cases between two medication groups; ${ }^{\dagger}$ comparison of the percentage of patients with rescue therapy between two medication groups; ${ }^{\ddagger}$ comparison of the percentage of patients receiving nasal glucocorticoids between two medication groups.

group in subjects with CRSsNP and eosinophilic CRSwNP at any of the follow-up visits (Figures S5 and S7). Patients with fluticasone propionate treatment demonstrated higher scores of discharge and edema at 3 and/or 6-month visit in non-eosinophilic CRSwNP group and whole CRS group, but lower scores of scarring at 3-month visit in non-eosinophilic CRSwNP group, compared with those treated with clarithromycin; however, no significant difference was discovered between two medications at 12-month follow-up visit in non-eosinophilic CRSwNP group and whole CRS group (Figures S6 and S8 in the Online Supplement).

Percentage of patients receiving nasal glucocorticoids and rescue oral steroid therapy after stop of study medication, and refractory cases

We compared the difference in percentages of patients receiving nasal glucocorticoids and rescue oral prednisone therapy after stop of study medication and patients with refractory CRS between fluticasone propionate and clarithromycin treatment group in whole CRS group and each subtype of CRS, and found no significant difference between two different treatment groups (Table 2). We also compared the numbers of oral steroid courses and the time from the 3-month follow-up visit (i.e. the time of stop of investigated medication) to the first course of rescue therapy in patients who received rescue therapy between two medication treatment groups, and found no significant difference either (Tables 3 and 4).

\section{Adverse events}

No serious adverse events were reported during the study. One patient in fluticasone propionate and two patients in clarithromycin group reported mild diarrhea. Headache was reported
Table 3. Numbers of oral steroid courses.

\begin{tabular}{|lccc|}
\hline & $\begin{array}{c}\text { Fluticasone pro- } \\
\text { pionate group }\end{array}$ & $\begin{array}{c}\text { Clarithromycin } \\
\text { group }\end{array}$ & P \\
\hline CRSsNP & $0(0-1)$ & $0(0-1)$ & 0.99 \\
\hline Non-Eos CRSWNP & $1(0-1.5)$ & $1(0-1)$ & 0.28 \\
\hline Eos CRSwNP & $0.5(0-1.25)$ & $1(0-2)$ & 0.91 \\
\hline Total CRS & $0.5(0-1)$ & $0(0-1)$ & 0.57
\end{tabular}

Results are expressed as medians and interquartile ranges. CRSsNP, chronic rhinosinusitis without nasal polyps; Non-Eos CRSwNP, noneosinophilic chronic rhinosinusitis with nasal polyps; Eos CRSwNP, eosinophilic chronic rhinosinusitis with nasal polyps; CRS, chronic rhinosinusitis. $\mathrm{P}$ values, comparison of the numbers of oral steroid courses between two medication groups.

Table 4. Time from the 3-month follow-up visit (the time point of stop of study medications) to the first course of rescue therapy (weeks).

\begin{tabular}{|lccc|}
\hline Fluticasone pro- & $\begin{array}{c}\text { Clarithromycin } \\
\text { pionate group }\end{array}$ & P \\
\hline CRSsNP & $6(4-8)$ & $8(5-9)$ & 0.56 \\
\hline Non-Eos CRSwNP & $8(4-12)$ & $8(6-9)$ & 0.86 \\
\hline Eos CRSwNP & $6(4-12)$ & $8(4-12)$ & 0.98 \\
\hline Total CRS & $8(4-12)$ & $8(4-10)$ & 0.93 \\
\hline
\end{tabular}

Results are expressed as medians and interquartile ranges. CRSsNP, chronic rhinosinusitis without nasal polyps; Non-Eos CRSwNP, noneosinophilic chronic rhinosinusitis with nasal polyps; Eos CRSwNP, eosinophilic chronic rhinosinusitis with nasal polyps; CRS, chronic rhinosinusitis. $P$ values, comparison of time difference between two medication groups in every phenotypic CRS. 
in one patient in both medication groups. Three patients in fluticasone propionate and two patients in clarithromycin group reported mild nasal bleeding. There was no significant difference in the number of and types of adverse events between fluticasone propionate group and clarithromycin group.

\section{Discussion}

Development of phenotype-orientated therapeutic strategies is critical for the improvement of CRS treatment. Although glucocorticoids are more likely to act on eosinophilic CRS, whether their benefit in the immediate postoperative period will vary according to different phenotypes of CRS is unclear. The efficacy of long-term low-dose macrolides as a pre- and post-operative treatment for CRS remains controversial (2). Two double-blind, placebo-controlled studies conducted in Europe reported no significant benefit of macrolides for CRS as a pre-operative treatment or postoperative add-on treatment to nasal steroid, respectively ${ }^{(6,29)}$. However, no clear separation between CRSwNP and CRSsNP was made in those studies ${ }^{(6,29)}$. Another doubleblind, placebo-controlled study on CRSsNP patients without surgery demonstrated that long-term, low-dose macrolide treatment might only be efficient for CRSsNP patients without elevated serum IgE levels; nevertheless, those results must be discussed with caution given the limited statistical power for sub-stratification analysis ${ }^{(4)}$. In contrast, Varvyanskaya et al. reported that post-operative, long-term, and low dose macrolide treatment as an add-on therapy to nasal glucocorticoid was able to control eosinophilic inflammation and prevent early relapse of polyps in CRSwNP patients ${ }^{(35)}$. Therefore, it is important to identify the phenotypes of CRS that may respond to glucocorticoid and macrolide treatment.

In this study, surprisingly, we could not show significant difference in alleviating subjective symptoms and promoting wound healing between fluticasone propionate and clarithromycin for CRSsNP and eosinophilic and non-eosinophilic CRSwNP patients after surgery. This finding is consistent with our previous ex vivo study showing comparable anti-inflammatory effects of clarithromycin and glucocorticoid in different phenotypic CRS (8). We found that both fluticasone propionate and clarithromycin could ameliorate the expression of chemokines for neutrophils, eosinophils, and Th2 cells in tissue explants ${ }^{(8)}$. Several studies have suggested a predominant role of long-term low-dose macrolide therapy in controlling neutrophil chemokine expression and neutrophilic inflammation ${ }^{(36)}$. However, recently, an effect of macrolide therapy in suppressing eosinophilic inflammation in patients with CRSwNP after surgery has also been demonstrated (35). On the other hand, neutrophils are generally considered to be less responsive to glucocorticoids. Nevertheless, there are some studies demonstrating a benefit of glucocorticoids for neutrophil inflammation. Vagaggini et al. found that inhaled budesonide was able to blunt the airway neutrophilic inflammati- on after ozone exposure ${ }^{(37)}$. Wang et al. provided direct evidence that glucocorticoids reduced IL-8 production in neutrophils from asthmatics in vitro ${ }^{(38)}$. Although our previous study on CRSwNP patients without surgery showed that oral glucocorticoids were less effective for those with neutrophilic inflammation, our current randomized controlled study with a larger sample size did not show significant difference of effect between fluticasone propionate and clarithromycin for non-eosinophilic CRSWNP patients after ESS. Given the change of inflammation load and anatomical structures of sinuses after surgery, the efficacy of anti-inflammatory medications may differ for CRS patients with and without surgery ${ }^{(25,39)}$.

We have to acknowledge several limitations of our current study. First, this is a single-blind trial without placebo control. The lack of placebo group makes our study unable to give a clear differentiation between the efficacy of medications and surgery. However, previous studies have already demonstrated the benefit of topical steroids over placebo on CRS in non-Chinese patients after ESS ${ }^{(25,40)}$. Thus, the effect of fluticasone propionate observed in this study, particularly for eosinophilic CRSWNP, unlikely merely attributes to the benefit of the ESS. Importantly, it should be noted that the lack of placebo group might increase the risk of a type 2 error. In addition, although we calculated the sample size for this study based on a pilot study following generally accepted protocols, the present study might be lack of power to detect the intervention effect, particularly for the subgroup analyses. Therefore, our data should be explained with caution and a multicenter study with larger sample size is required to confirm our current findings. Second, again, due to the ethical consideration, the additional visits to physicians and rescue therapy were allowed in our study. The high frequency of usage of rescue therapy may obscure the comparison of clarithromycin and fluticasone propionate. However, the comparable rescue therapy usage in clarithromycin and fluticasone propionate group supports our conclusion. Third, in this study, we only evaluated the changes of subjective symptoms and objective endoscopic appearance, a more comprehensive assessment of quality of life, nasal resistance, and olfactory function would help to delineate the effect of fluticasone propionate and clarithromycin more precisely. Fourth, in this study, we defined eosinophilic CRSwNP based on our previously published cut-off ${ }^{(11)}$. Although distinct immunopathologic and clinical characteristics have been demonstrated for non-eosinophilic and eosinophilic CRSwNP classified by our cut-off ${ }^{(8,11,12,25)}$, we cannot rule out the possibility that different post-operative effects may be observed for glucocorticoids and macrolides if CRSwNP was sub-grouped by a different cut-off for eosinophilic inflammation. Fifth, we cannot fully preclude the potential floor effect either. Nevertheless, we want to emphasize that we performed sample size calculation following generally accepted statistical analysis protocol ${ }^{(36)}$. Sixth, we set 3 months as treatment time period for 
both medications. However, whether longer treatment duration and combination of two medications will have improved effect deserve further investigations. These comments notwithstanding, our present study is the first randomized controlled trial comparing the post-operative efficacy of glucocorticoid versus macrolide in different subtypes of CRS defined based on our current phenotyping strategy.

\section{Conclusion}

We did not show significant difference between these two medications. Given the potential risk of development of resistance to macrolides after long-term usage and relatively higher cost of macrolides compared to intranasal steroid sprays ${ }^{(41)}$, there is no justification for giving post-operative macrolides routinely instead of a simple intranasal steroid spray.

\section{Acknowledgement}

This study was supported by National Natural Science Foundation of China (NSFC) grants [81325006, 81570899 and 81630024 (Z.L.), 81200733 (H.W.), 81400448 (X.B.L), and 81400449 (P.P.C.)], Hubei Province Natural Science Foundation grant 2017 CFA016 (Z.L.), and Program for Changjiang Scholars and Innovative Research Team in University [IRT_14R20].

\section{Authorship contribution}

MZ designed the study, analyzed data, and prepared manuscript; HW participated in subject enrollment and manuscript preparation; $\mathrm{BL}, \mathrm{HW}$, and XBL collected surgical samples; JM and JXL participated in symptom severity record; PPC and QN participated in data discussion; ZL designed the study, did endoscopic examination and surgery, and prepared the manuscript.

\section{Conflict of interest}

No conflict of interest.

\section{References}

1. Shi JB, Fu QL, Zhang H, et al. Epidemiology of chronic rhinosinusitis: results from a cross-sectional survey in seven Chinese cities. Allergy 2015; 70:533-539.

2. Bachert C, Zhang L, Gevaert P. Current and future treatment options for adult chronic rhinosinusitis: Focus on nasal polyposis. Allergy Clin Immunol 2015; 136:1431-1440.

3. Fokkens WJ, Lund VJ, Mullol J, et al. European position paper on rhinosinusitis and nasal polyps. Rhinol Suppl 2012; 23 : 1-298.

4. Wallwork B, Coman W, Mackay-Sim A, Greiff L, Cervin A. A double-blind, randomized, placebo-controlled trial of macrolide in the treatment of chronic rhinosinusitis. Laryngoscope 2006; 116:189-193.

5. Pinto Bezerra Soter AC, Bezerra TF, Pezato $\mathrm{R}$, et al. Prospective open-label evaluation of long-term low-dose doxycycline for difficult-to-treat chronic rhinosinusitis with nasal polyps. Rhinology. 2017;55:175-180.

6. Videler WJ, Badia L, Harvey RJ, et al. Lack of efficacy of long-term, low-dose azithromycin in chronic rhinosinusitis: a randomized controlled trial. Allergy 2011; 66:1457-1468.

7. Tomassen P, Vandeplas G, Van Zele T, et al. Inflammatory endotypes of chronic rhinosinusitis based on cluster analysis of biomarkers. J Allergy Clin Immunol 2016; 137:14491456

8. Zeng M, Li ZY, Ma J, et al. Clarithromycin and dexamethasone show similar antiinflammatory effects on distinct phenotypic chronic rhinosinusitis: an explant model study. BMC Immunol 2015; 16:37.

9. Lou H, Meng Y, Piao Y, et al. Cellular phenotyping of chronic rhinosinusitis with nasal polyps. Rhinology. 2016:54:150-159.

10. Zhang N, Van Zele T, Perez-Novo C, et al Different types of T-effector cells orchestrate mucosal inflammation in chronic sinus disease. J Allergy Clin Immunol 2008; 122:961-968.

11. Cao PP, Li HB, Wang BF, et al. Distinct immunopathologic characteristics of various types of chronic rhinosinusitis in adult Chinese. J Allergy Clin Immunol 2009; 124:478-484.

12. Shi LL, Song J, Xiong P, et al. Disease-specific T-helper cell polarizing function of lesional dendritic cells in different types of chronic rhinosinusitis with nasal polyps. Am J Respir Crit Care Med 2014; 190: 628-638.

13. Zhang $Y N$, Song J, Wang $H$, et al. Nasa IL-4+CXCR5+CD4+ T follicular helper cell counts correlate with local lgE production in eosinophilic nasal polyps. J Allergy Clin Immunol 2016; 137:462-473.

14. Derycke L, Eyerich S, Van Crombruggen $K$, et al. Mixed $T$ helper cell signatures in chronic rhinosinusitis with and without polyps. PLoS One 2014; 9: e97581.

15. Peters AT, Kato A, Zhang N, et al. Evidence for altered activity of the IL- 6 pathway in chronic rhinosinusitis with nasal polyps. Allergy Clin Immunol 2010; 125:397-403.

16. Wen W, Liu W, Zhang L, et al. Nasal Health Group, China (NHGC): Increased neutrophilia in nasal polyps reduces the response to oral corticosteroid therapy. J Allergy Clin Immunol 2012; 129:1522-1528.

17. Luo Q, Chen F, Liu W, et al. Nasal Health Group, China (NHGC): evaluation of long-term clarithromycin treatment in adult Chinese patients with chronic rhinosinusitis without nasal polyps. ORL J Otorhinolaryngol Relat Spec 2011; 73:206211.

18. Orlandi RR, Smith TL, Marple BF, et al. Update on evidence-based reviews with recommendations in adult chronic rhinosinusitis. Int Forum Allergy Rhinol 2014; 4:S1S15.

19. Orlandi RR, Kingdom TT, Hwang PH, et al
International Consensus Statement on Allergy and Rhinology: Rhinosinusitis. Int Forum Allergy Rhinol 2016; 6 Suppl 1:S22209.

20. Bousquet J, Schünemann HJ, Samolinski $B$, et al. Allergic Rhinitis and its Impact on Asthma (ARIA): achievements in 10 years and future needs. J Allergy Clin Immunol 2012; 130:1049-1062.

21. Volbeda F, Broekema M, Lodewijk ME, et al. Clinical control of asthma associates with measures of airway inflammation. Thorax 2013; 68:19-24.

22. Hu Y, Cao PP, Liang GT Cui YH, Liu Z. Diagnostic significance of blood eosinophil count in eosinophilic chronic rhinosinusitis with nasal polyps in Chinese adults. Laryngoscope 2012; 122:498-503.

23. Stammberger $H$. Endoscopic surgery for mycotic and chronic recurring sinusitis. Ann Otol Rhinol Laryngol Suppl 1985; 119:1-11.

24. Kennedy DW. Functional endoscopic sinus surgery. Technique. Arch Otolaryngol 1985; 111: 643-649.

25. Jorissen M, Bachert C. Effect of corticosteroids on wound healing after endoscopic sinus surgery. Rhinology 2009; 47:280-286.

26. Lund VJ, Kennedy DW. Staging for rhinosinusitis. Otolaryngol Head Neck Surg 1997; 117: $\$ 35-40$

27. Small CB, Hernandez J, Reyes A, et al. Efficacy and safety of mometasone furoate nasal spray in nasal polyposis. J Allergy Clin Immunol 2005;116: 1275-1281.

28. Ozturk F, Bakirtas A, Ileri F, Turktas I. Efficacy and tolerability of systemic methylprednisolone in children and adolescents with chronic rhinosinusitis: a double-blind, placebo-controlled randomized trial. J Allergy Clin Immunol 2011;128:348-352.

29. Haxel BR, Clemens M, Karaiskaki N, Dippold U, Kettern L, Mann WJ. Controlled trial for long-term low-dose erythromycin after 
sinus surgery for chronic rhinosinusitis. Laryngoscope 2015; 125:1048-1055.

30. Rowe-Jones JM, Medcalf M, Durham SR, Richards DH, Mackay IS. Functional endoscopic sinus surgery: 5 year follow up and results of a prospective, randomised, stratified, double-blind, placebo controlled study of postoperative fluticasone propionate aqueous nasal spray. Rhinology 2005; 43:210.

31. Zeng M, Long XB, Cui YH, Liu Z. Comparison of efficacy of mometasone furoate versus clarithromycin in the treatment of chronic rhinosinusitis without nasal polyps in Chinese adults. Am J Rhinol Allergy 2011; 25:e203-207.

32. Zhang L, Zhang LH. Comparison of different endoscopic scoring systems in patients with chronic rhinosinusitis: reliability, validity, responsiveness and correlation. Rhinology 2017;55:363-368.

33. McCoul ED, Tabaee A. A Practical Approach to Refractory Chronic Rhinosinusitis. Otolaryngol Clin North Am 2017;50:183198.

34. Angst F, Aeschlimann A, Stucki G. Smallest detectable and minimal clinically important differences of rehabilitation intervention with their implications for required sample sizes using WOMAC and SF-36 quality of life measurement instruments in patients with osteoarthritis of the lower extremities. Arthritis Rheum 2001;4:384-391.

35. Varvyanskaya A, Lopatin A. Efficacy of longterm low-dose macrolide therapy in preventing early recurrence of nasal polyps after endoscopic sinus surgery. Int Forum Allergy Rhinol 2014; 4:533-541.

36. Simpson JL, Powell H, Boyle MJ, Scott RJ, Gibson PG. Clarithromycin targets neutrophilic airway inflammation in refractory asthma. Am J Respir Crit Care Med 2008;177:148-155.

37. Vagaggini B, Taccola M, Conti I, et al. Budesonide reduces neutrophilic but not functional airway response to ozone in mild asthmatics. Am J Respir Crit Care Med 2001;164:2172-2176.

38. Wang M, Gao P, Wu X, et al. Impaired antiinflammatory action of glucocorticoid in neutrophil from patients with steroid-resistant asthma. Respir Res 2016;17:153.

39. Watelet JB, Demetter P, Claeys C, Cauwenberge $P$, Cuvelier $C$, Bachert $C$. Wound healing after paranasal sinus surgery: neutrophilic inflammation influences the outcome. Histopathology 2006:48:174181.

40. Fandiño M, Macdonald KI, Lee J, Witterick IJ. The use of postoperative topical corticos- teroids in chronic rhinosinusitis with nasal polyps: a systematic review and meta-analysis. Am J Rhinol Allergy 2013;27:e146-157

41. Altenburg J, de Graaff CS, van der Werf TS, Boersma WG. Immunomodulatory effects of macrolide antibiotics - part 2: advantages and disadvantages of long-term, low-dose macrolide therapy. Respiration 2011;81:7587.

Zheng Liu, MD., Ph.D.

Department of Otolaryngology-Head and Neck Surgery

Tongji Hospital, Tongji Medical

College

Huazhong University of Science and

Technology

No. 1095 Jiefang Avenue

Wuhan 430030

P.R.China

E-mail: zhengliuent@hotmail.com

\section{ADVERTISEMENT}

\section{6th INTERNATIONAL}

COURSE IN ADVANCED SINUS SURGERY TECHNIQUES

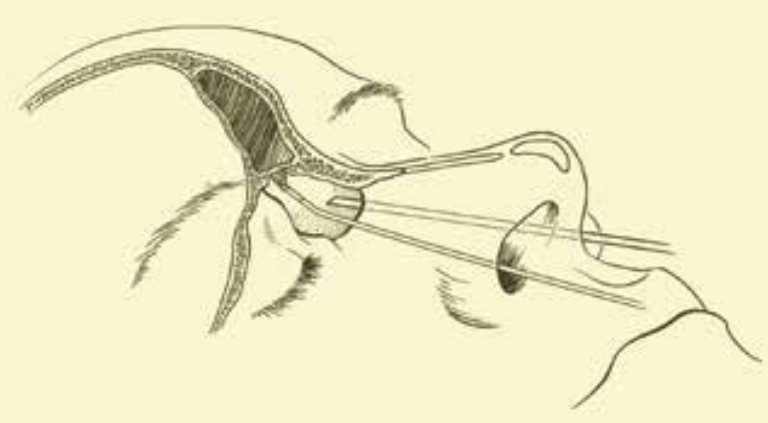

28-29-30 October 2019

Department of Otorhinolaryngology Academic Medical Center of the University of Amsterdam The Netherlands

For further information Email:secretariaatkno@amc.uva.nl Web: www.sinuscourse.nl 